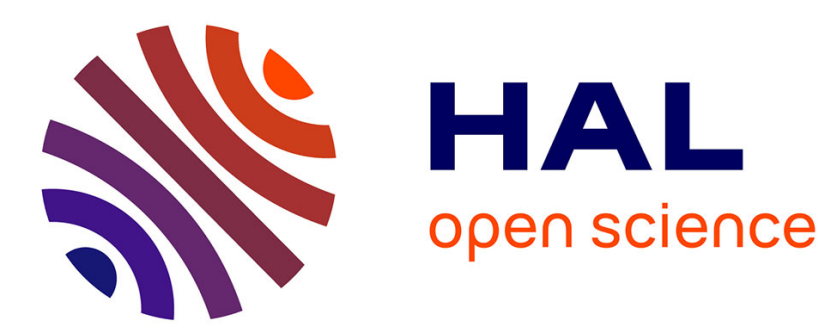

\title{
Expectations about motion direction affect perception and anticipatory smooth pursuit differently
}

Xiuyun Wu, Austin C Rothwell, Miriam Spering, Anna Montagnini

\section{To cite this version:}

Xiuyun Wu, Austin C Rothwell, Miriam Spering, Anna Montagnini. Expectations about motion direction affect perception and anticipatory smooth pursuit differently. Journal of Neurophysiology, 2021, 10.1101/2020.11.02.365338. hal-03415471

\section{HAL Id: hal-03415471 \\ https://hal.science/hal-03415471}

Submitted on 4 Nov 2021

HAL is a multi-disciplinary open access archive for the deposit and dissemination of scientific research documents, whether they are published or not. The documents may come from teaching and research institutions in France or abroad, or from public or private research centers.
L'archive ouverte pluridisciplinaire HAL, est destinée au dépôt et à la diffusion de documents scientifiques de niveau recherche, publiés ou non, émanant des établissements d'enseignement et de recherche français ou étrangers, des laboratoires publics ou privés. 


\section{Expectations about motion direction affect perception and anticipatory smooth pursuit differently}

Xiuyun $\mathrm{Wu}^{1,2 *}$, Austin C. Rothwell ${ }^{2}$, Miriam Spering ${ }^{2,3,4}, \&$ Anna Montagnini ${ }^{5}$

${ }^{1}$ Graduate Program in Neuroscience, University of British Columbia, Vancouver, BC, Canada

${ }^{2}$ Department of Ophthalmology \& Visual Sciences, University of British Columbia, Vancouver, BC, Canada

${ }^{3}$ Djavad Mowafaghian Center for Brain Health, University of British Columbia, Vancouver, BC, Canada

${ }^{4}$ Institute for Computing, Information and Cognitive Systems, University of British Columbia, Vancouver, BC, Canada

${ }^{5}$ Institut de Neurosciences de la Timone, CNRS and Aix-Marseille Université, Marseille, France

* Corresponding author's contact:

xiuyun.wu@student.ubc.ca 


\section{Abstract}

2 Smooth pursuit eye movements and visual motion perception rely on the integration of current

3 sensory signals with past experience. Experience shapes our expectation of current visual events

4 and can drive eye movement responses made in anticipation of a target, such as anticipatory

5 pursuit. Previous research revealed consistent effects of expectation on anticipatory pursuit-eye

6 movements follow the expected target direction or speed — and contrasting effects on motion

7 perception, but most studies considered either eye movement or perceptual responses. The

8 current study directly compared effects of direction expectation on perception and anticipatory

9 pursuit within the same direction discrimination task to investigate whether both types of

10 responses are affected similarly or differently. Observers $(n=10)$ viewed high-coherence

11 random-dot kinematograms (RDKs) moving rightward and leftward with a probability of 50, 70,

12 or $90 \%$ in a given block of trials to build up an expectation of motion direction. They were asked

13 to judge motion direction of interleaved low-coherence RDKs (0-15\%). Perceptual judgements

14 were compared to changes in anticipatory pursuit eye movements as a function of probability.

15 Results show that anticipatory pursuit velocity scaled with probability and followed direction

16 expectation (attraction bias), whereas perceptual judgments were biased opposite to direction

17 expectation (repulsion bias). Control experiments suggest that the repulsion bias in perception

18 was not caused by retinal slip induced by anticipatory pursuit, or by motion adaptation. We

19 conclude that direction expectation can be processed differently for perception and anticipatory

20 pursuit. 


\section{Introduction}

How we perceive and interact with the visual world depends not only on current visual

23 input but also on our experience with past sensory events. In Bayesian inference, this experience

24 informs a prior - one's expectation of the probability of an event before any sensory evidence is

25 present (de Lange et al. 2018; Seriès and Seitz 2013). This study investigates how visual motion

26 priors, based on long-term experience, affect visual perception and movement, and whether these

27 two outcomes are controlled in the same way or differently by expectation. We use smooth

28 pursuit eye movements - the eyes' continuous response to moving objects — as a model system

29 for visually-guided movement to investigate this question. Smooth pursuit eye movements are

30 closely related to the perception of visual motion (Gegenfurtner 2016; Schütz et al. 2011;

31 Spering and Montagnini 2011). They rely on the integration of current motion information with

32 priors based on experience across just a few trials or across a longer-term context (Darlington et

33 al. 2017; Deravet et al. 2018; Yang et al. 2012). Moreover, smooth pursuit can be triggered by the

34 expectation of a certain motion direction even before the object's motion onset, a phenomenon

35 known as anticipatory smooth pursuit (Kowler et al. 1984, 2019).

36 Previous research has revealed highly consistent effects of expectation on pursuit but

37 contrasting effects on motion perception. For example, anticipatory pursuit can be triggered

38 when observers repeatedly view stimuli moving into the same direction (Kowler 1989; Kowler et

39 al. 2019). The eyes are then attracted to the expected motion direction prior to the onset of the

40 stimulus (attraction bias in direction). These responses are not purely habitual but finely tuned to

41 the strength of expectation (Damasse et al. 2018; Jarrett and Barnes 2002; Santos and Kowler

42 2017). Congruently, anticipatory pursuit velocity is proportional to the average velocity of the

43 target across previous trials (attraction bias in speed), and strongly affected by events in the 
44 previous two trials (Maus et al. 2015). Furthermore, Bayesian integration models have been used

45 to describe how priors would lead to attraction effects in visually-guided pursuit when combined

46 with noisy visual motion signals (Behling and Lisberger 2020; Darlington et al. 2017; Deravet et

47 al. 2018).

By contrast, perceptual studies have found evidence for both an attraction bias as well as

49 for responses to be repelled away from the expected direction (repulsion bias; Jazayeri and

50 Movshon 2007). In studies reporting attraction biases, estimates of direction, velocity, or

51 orientation in a current trial are affected by events in previous trials such that an observer's

52 perception would be biased in line with the motion information observed in previous trials. Such

53 biases can build up quickly, within a few trials (Alais et al. 2017; Cicchini et al. 2018) or can be

54 based on implicitly learning the statistical properties of a stimulus environment over many trials

55 (Chalk et al. 2010; Kok et al. 2013; Seriès and Seitz 2013), as described by Bayesian integration

56 models. Perceptual repulsion biases have been observed across different visual tasks and

57 features. In a speed estimation task in which observers had to judge whether the speed in the

58 current trial was faster or slower than the average speed across all previous trials, observers

59 tended to overestimate a current target's velocity when the average velocity across previous trials

60 was slow and vice versa for fast velocity (Maus et al. 2015). Similar repulsion biases have been

61 found in studies in which observers had to adjust the orientation of a test stimulus relative to an

62 inducer stimulus, when both stimulus orientations differed by more than $60^{\circ}$ between the

63 previous and current trial (Fritsche et al. 2020). In this scenario, observers' adjustment responses

64 were sometimes repelled away from the previous trial's stimulus orientation. In sum, expectation

65 built across different timescales can result in a perceptual bias either in the same direction as the

66 cue, prompt, or adaptor (attraction bias), or in the opposite direction (repulsion bias). 
The current study directly compared effects of direction expectation on perception and

68 anticipatory pursuit within the same trials to investigate whether both types of responses are

69 affected similarly or differently, and how they interact. On one hand, attraction biases are

70 commonly found in pursuit, and in most perceptual studies that did not use adjustment tasks or

71 reference comparisons. On the other hand, one study that directly compared velocity expectation

72 effects reported opposite biases in speed discrimination and anticipatory pursuit (Maus et al.

73 2015). Overall, it remains unclear whether motion priors affect perception and pursuit similarly

74 or differently. We introduced different probabilities of motion direction in the current study,

75 leading to an implicit expectation bias for future motion direction based on previous trial history.

76 This manipulation allows us to investigate the effect of a general motion prior on perception and

77 pursuit. In the following, we refer to effects of expectation as the behavior triggered by

78 manipulations of this statistical bias.

\section{Methods}

All three experiments were similar in terms of procedure and analyses. Experiment 1 was

82 the main experiment, and the purpose was to compare the effect of expectation on motion

83 direction discrimination and anticipatory pursuit. Control experiments 2 and 3 investigated

84 alternative explanations for findings obtained in Experiment 1, testing interactions between

85 anticipatory pursuit and perception, and effects of stimulus features, respectively. General

86 methods are described for experiment 1. Deviations in stimuli and procedures for control

87 experiments are briefly described in Results.

88

89 Observers 
We recruited 10 observers (age $M=26.20$, std $=5.41$ years; six females) with normal or

91 corrected-to-normal visual acuity (at least 20/20 as assessed using an Early Treatment Diabetic

92 Retinopathy Study chart) and no history of ophthalmologic, neurologic, or psychiatric disease.

93 All observers participated in experiment 1, eight of these observers (age $M=27.38$, std $=5.40$

94 years; four females) also participated in experiment 2, and nine (age $M=26.56$, std $=5.61$ years;

95 six females) also participated in experiment 3. The sample size is comparable to previous studies

96 ( $n=9$ in Maus et al. 2015; $n=8$ in Santos et al. 2012; $n=6$ in Santos and Kowler 2017). The

97 University of British Columbia Behavioral Research Ethics Board approved all experimental

98 procedures, and all observers participated after giving written informed consent. Observers

99 received \$35 Canadian Dollars remuneration for participation per experiment.

100

Visual stimuli and setup

102

Stimuli were random dot kinematograms $(\mathrm{RDKs})$ presented in a static aperture of $20^{\circ}$

103 diameter centered in the middle of the screen. Each RDK consisted of about 470 (density 1.5

$\left.104 \mathrm{dot} / \mathrm{deg}^{2}\right)$ uniformly distributed white dots $\left(98 \mathrm{~cd} / \mathrm{m}^{2}\right)$ on a grey background $\left(22 \mathrm{~cd} / \mathrm{m}^{2}\right)$. Each dot

105 (diameter $0.14^{\circ}$ ) moved at a constant speed of $10^{\circ} / \mathrm{s}$. The dots were labeled as signal or noise

106 dots. Labels were updated and randomly reassigned every four frames (about 47 milliseconds,

$107 \mathrm{~ms}$ ). Signal dots always moved in the global motion direction of the RDK (left or right), while

108 each noise dot moved in a random direction other than the signal direction with unlimited

109 lifetime. When a dot moved out of the aperture, it reentered from the opposite side of the

110 aperture. The coherence of the RDK was defined as the proportion of signal dots $(0-100 \%)$.

111 Observers were seated in a dimly-lit room and viewed all stimuli on a gamma-corrected

$11239 \mathrm{~cm} \times 29 \mathrm{~cm}$ CRT monitor (ViewSonic G255f; resolution $1280 \times 1024$ pixel; refresh rate 85 
$113 \mathrm{~Hz}$ ). The viewing distance was $55 \mathrm{~cm}$. Each observer's head was stabilized using a chin-and-

114 forehead-rest. Stimuli and procedure were programmed in MATLAB R2018b (The MathWorks

115 Inc., Natick, MA) and Psychtoolbox Version 3.0.12 (Brainard 1997; Kleiner et al. 2007; Pelli

116 1997).

118 Procedure and design

119 Figure 1 shows the trial timeline in experiment 1. Observers were asked to fixate the

120 center of the screen when the red fixation point was on for 600-900 ms. Fixation was monitored

121 online: if eye position was further than $2^{\circ}$ from the center of the fixation point, the fixation point

122 turned white and the countdown of fixation duration was paused until the observer regained

123 accurate fixation. A blank screen (gap; $300 \mathrm{~ms}$ ) was shown to help induce anticipatory pursuit

124 (Krauzlis and Miles 1996). Observers were then asked to smoothly follow the global motion of

125 the RDK (700 ms) with their eyes. A dynamic white-noise mask with luminance noise randomly

126 assigned pixel by pixel (luminance range within $7 \mathrm{~cd} / \mathrm{m}^{2}$ to $46 \mathrm{~cd} / \mathrm{m}^{2}$ ) was shown after RDK

127 offset for $600 \mathrm{~ms}$ to reduce potential motion aftereffects. At the end of each trial, they were asked

128 to report whether it moved left or right using the "left" or "right" arrow keys on the computer

129 keyboard. 


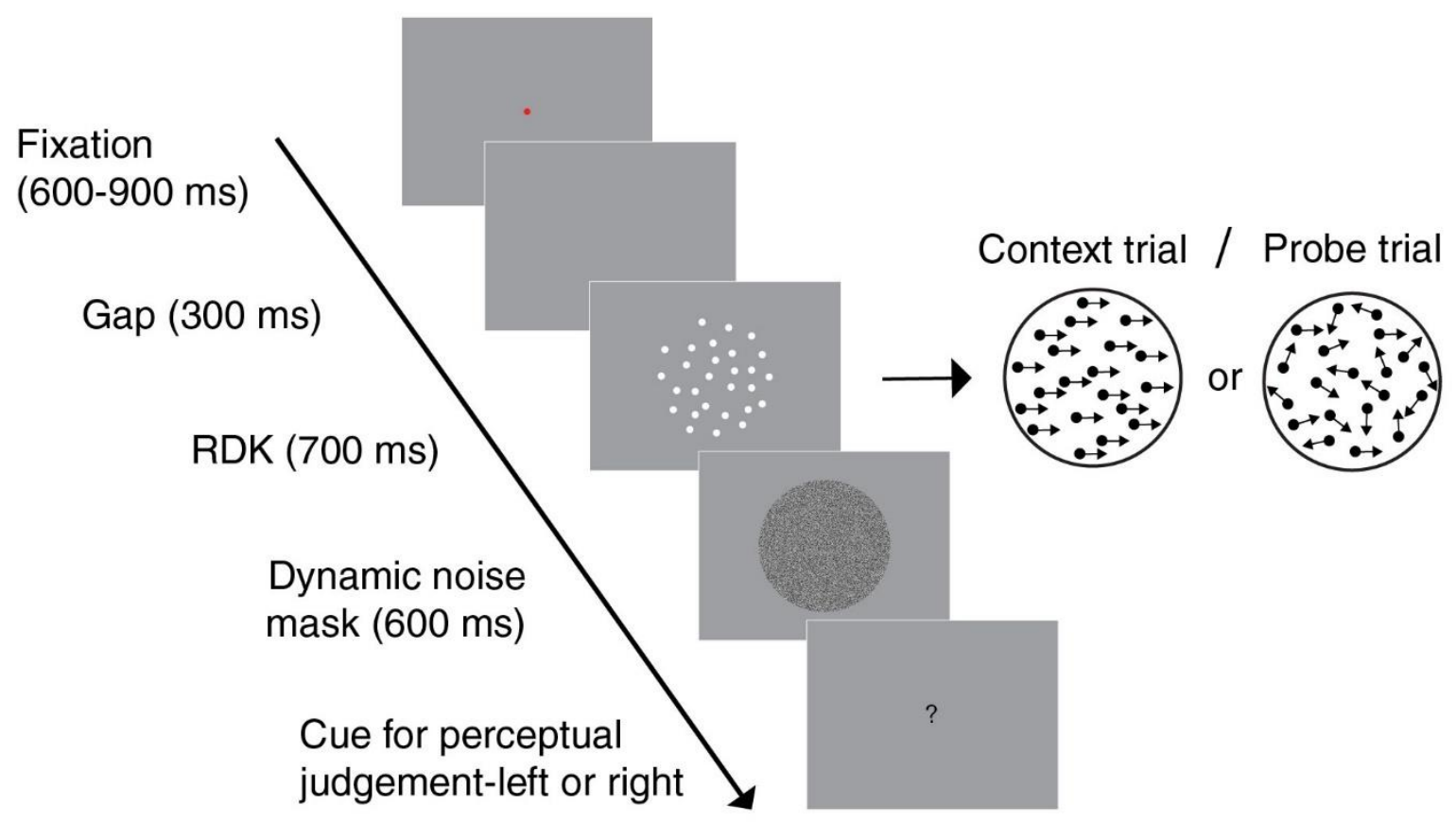

131 Figure 1. Trial timeline in experiment 1. A fixation point was shown for 600-900 ms, followed by a blank screen for $300 \mathrm{~ms}$, and the RDK for $700 \mathrm{~ms}$. A dynamic white-noise mask was

133 presented after the RDK for $600 \mathrm{~ms}$. Two types of trials were presented: context trials with

134 highest-coherence RDKs, and probe trials with low-coherence RDKs. The stimuli's relative size and contrast are increased for presentation purposes.

In each block, two types of trials were shown: 500 context trials containing non-

138 ambiguous motion direction (100\% coherence) were randomly interleaved with 182 probe trials

139 containing ambiguous motion $(0,5,10$ or $15 \%$ coherence). The purpose of the context trials was

140 to build up an expectation of motion direction in a given block. In each of three blocks of trials,

141 presented in random order, we introduced different probabilities of motion direction in context

142 trials, either $50 \%, 70 \%$, and $90 \%$ probability of rightward or leftward motion. Blocks with higher

143 probability of rightward and leftward motion were presented in different sessions; half of our

144 sample of observers $(n=5)$ saw only higher probabilities of rightward motion, the other half saw

145 only higher probabilities of leftward motion. The experiment was split up that way to reduce

146 workload for each observer, because each block of trials took 60 minutes to complete for a total

147 of 3 hours per observer. The first 50 trials in each block were always context trials. The purpose 
148 of the probe trials was to measure the effect of expectation on perception of motion direction,

149 which would be prominent when visual input provided little evidence. In order to fairly compare

150 perception and oculomotor anticipation we also analyzed anticipatory pursuit in probe trials only.

151 For all observers, probe trials consisted of equal numbers of leftward/rightward trials.

\section{Eye movement recording and analysis}

In all three experiments, the position of the right eye was recorded using a video-based

155 eye tracker at a sampling rate of $1000 \mathrm{~Hz}$ (EyeLink 1000 desk-mounted, SR Research Ltd.,

156 Kanata, ON, Canada). Eye movements were then analyzed offline using custom-made MATLAB

157 functions. Eye position, velocity, and acceleration data were filtered with a second-order

158 Butterworth filter (cutoff frequencies of $15 \mathrm{~Hz}$ for position and $30 \mathrm{~Hz}$ for velocity and

159 acceleration). Saccades were detected based on an acceleration criterion: the acceleration trace

160 was segmented by zero-crossing points, and peak acceleration within each segment was

161 calculated. If at least two consecutive segments had absolute peak acceleration larger than

$162400^{\circ} / \mathrm{s}^{2}$, these segments were defined as saccades. An acceleration threshold was used to

163 accurately detect saccades of small amplitude and velocity during the anticipatory pursuit phase.

164 Saccade detection was confirmed by visual inspection of the velocity traces in each trial.

165 Saccades were then excluded from the analysis of smooth pursuit. Following previous studies

166 (Maus et al. 2015; Santos and Kowler 2017; Watamaniuk et al. 2017), anticipatory pursuit

167 velocity was defined as the average horizontal eye velocity during the time window from $50 \mathrm{~ms}$

168 before to $50 \mathrm{~ms}$ after RDK onset. We also analyzed eye velocity gain (eye velocity relative to

169 target velocity) during visually-guided pursuit, calculated during the time window from 300 to

$170600 \mathrm{~ms}$ after target onset. Trials with blinks during RDK presentation were manually labeled as 
171 invalid and excluded (1\% across observers in experiment $1,0.5 \%$ in experiment 2 , and $0.7 \%$ in

172 experiment 3). Leftward direction is negative by convention.

173

174 Perceptual response analysis

175 We did not observe systematic differences between the effects of rightward and leftward

176 motion probability on the magnitude of anticipatory pursuit (experiment $1: t(4)=0.76, p=.49$,

177 Cohen's $d=0.94$ ) or perceptual bias (experiment 1:t(4)=1.07, $p=.34$, Cohen's $d=0.83$ ) and

178 therefore merged data for different motion directions, presenting data as if higher probabilities of

179 rightward motion were presented. Under each probability condition for each observer, we fitted a

180 psychometric curve using the logistic function as shown below:

181

$P($ perceiving right $)=(1-\lambda) \frac{1}{1+e^{-\beta(x-\alpha)}}$

Specifically, $x$ is the signed motion coherence of RDK (negative for leftward motion), $\alpha$

184 is the point of subjective equality (PSE) where observers reported both motion directions equally

185 often ( $50 \%$ of the time), $\beta$ is the slope representing the sensitivity of perception, and $\lambda$ is the

186 lapse rate (restricted to below 0.1 when fitting). In this study, a shift of the PSE across

187 probability conditions would indicate a perceptual bias. A shift to the left indicates a perceptual

188 attraction bias (i.e. with direction judgments being attracted toward the direction expectation of

189 rightwards), and a shift to the right indicates a repulsion bias. A change in slope across

190 probability conditions would indicate a change in sensitivity of perceptual judgments, where a

191 steeper slope corresponds to higher sensitivity. Curve fitting was performed using the Palamedes

192 toolbox version 1.9.0 in MATLAB (Prins and Kingdom 2018). 
Hypotheses and statistical analysis

In experiment 1 , we aimed to test the following hypotheses. First, anticipatory pursuit is

196 affected by direction expectation: the velocity of anticipatory pursuit scales positively with the

197 target's direction probability (attraction bias); a higher probability of rightward motion will lead

198 to a higher velocity in anticipatory pursuit (Fig. 2a). Second, direction perception is affected by

199 direction expectation: observers preferentially perceive the expected motion direction (attraction

200 bias; Fig. 2b). Alternatively, perception could be biased away from the expected direction

201 (repulsion bias; Fig. 2b), as has sometimes been reported in the literature. We further examined

202 if expectation affected slope to investigate whether different prior probabilities might result in

203 differences in sensitivity. To examine the expected effects of probability on anticipatory pursuit

204 velocity, the magnitude of perceptual bias (shift of the PSE), and the sensitivity of perception

205 (slope), we used one-way repeated-measures analyses of variance (ANOVA) with probability as

206 factor. In addition, to examine whether anticipatory pursuit velocity and the strength of any

207 potential perceptual bias were correlated across conditions and observers, we fitted a linear

208 mixed-effects model of PSE with probability and anticipatory pursuit velocity as fixed effects,

209 and individual intercept as the random effect (formula: PSE anticipatory pursuit velocity +

210 probability $+(1 \mid$ observer $))$. Finally, we also examined the potential link between visually-

211 guided pursuit and perception, and effects of probability on the velocity gain of visually-guided

212 pursuit. Experiments 2 and 3 investigated alternative explanations of findings obtained in

213 experiment 1 . Their logic and underlying hypotheses are described in Results. 


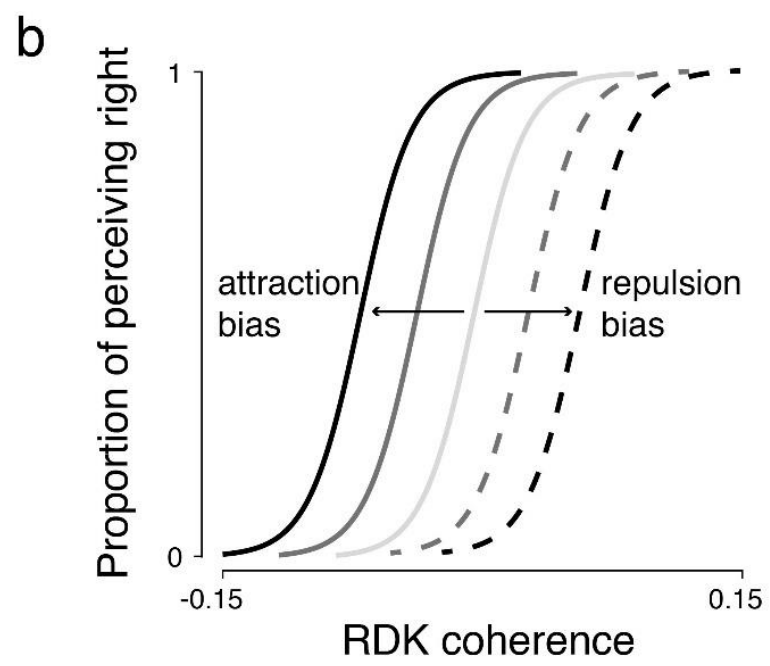

Figure 2. Hypotheses for (a) attraction bias in anticipatory pursuit velocity and (b) attraction or repulsion bias in perception. (a) Anticipatory pursuit velocity increases with increasing probability of rightward motion in each block, reflecting an attraction bias. (b) The perceptual bias is reflected by a shift of the PSE at higher probabilities (70\% and 90\%) when compared to the $50 \%$ probability condition; a leftward shift (solid lines) indicates an attraction bias, a rightward shift (dashed lines) indicates repulsion bias. Negative value of RDK coherence indicates that the global motion direction is left.

Across experiments, we report generalized eta-squared $\left(\eta_{g}^{2}\right)$ as the effect size in one-way ANOVAs, and partial eta-squared $\left(\eta_{p}^{2}\right)$ in two-way ANOVAs. For all experiments, we also report mean and 95\% confidence interval (CI) of anticipatory pursuit velocity, and PSE from 1000 bootstrap simulations to supplement statistical hypothesis testing and provide quantitative estimates of the variability of sample estimates. The statistical tests were conducted in R Version 3.6.0 (package "lme4", Bates et al. 2015; package "ez", Lawrence 2016; R Core Team 2019) and MATLAB R2020a.

\section{Experiment 1}

\section{Evidence for attraction bias in anticipatory pursuit}

Across observers and trials, we found that the velocity of the anticipatory pursuit 
236 response scaled positively with the probability of a given motion direction. Figure 3a shows an

237 example of individual eye velocity traces and Figure 3b shows group-averaged eye velocity

238 traces in probe trials. indicating that anticipatory pursuit velocity increases with increasing

239 probability of rightward motion. These observations were confirmed by a significant main effect

240 of probability on anticipatory pursuit velocity $(\mathbf{F i g} . \mathbf{3 c}), F(2,18)=28.19, p=2.84 \times 10^{-6}, \eta_{g}^{2}=.19$.

241 As a complementary method to these statistics, the bootstrapped mean and 95\% CI of

242 anticipatory pursuit velocity confirmed our findings $(50 \%: 0.24 \pm 0.01 \%$ s; $70 \%: 0.70 \pm 0.01 \%$;

$24390 \%: 1.26 \pm 0.01 \%$ ) 


\section{a}

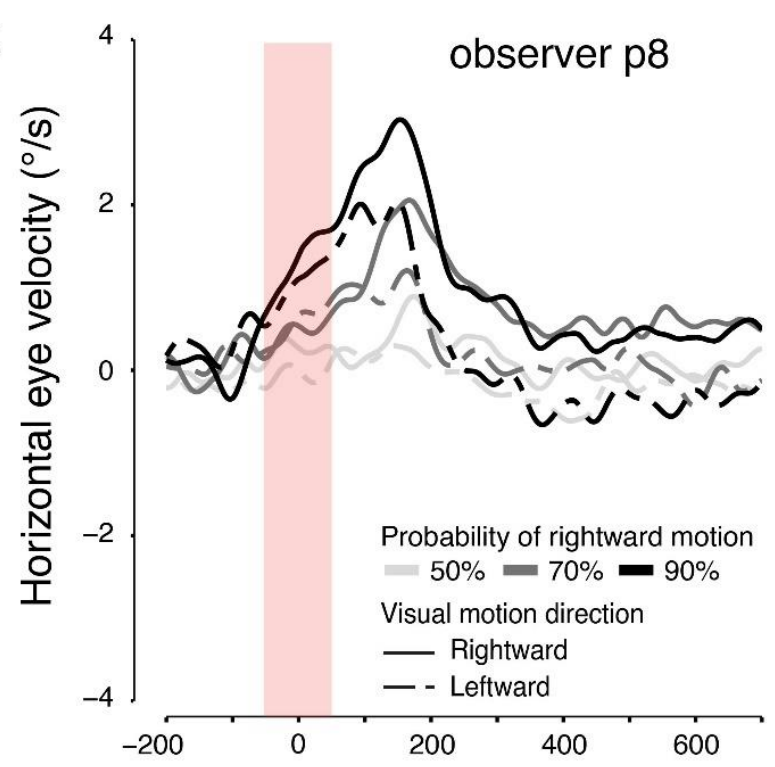

$\mathrm{b}^{4} \quad n=10$

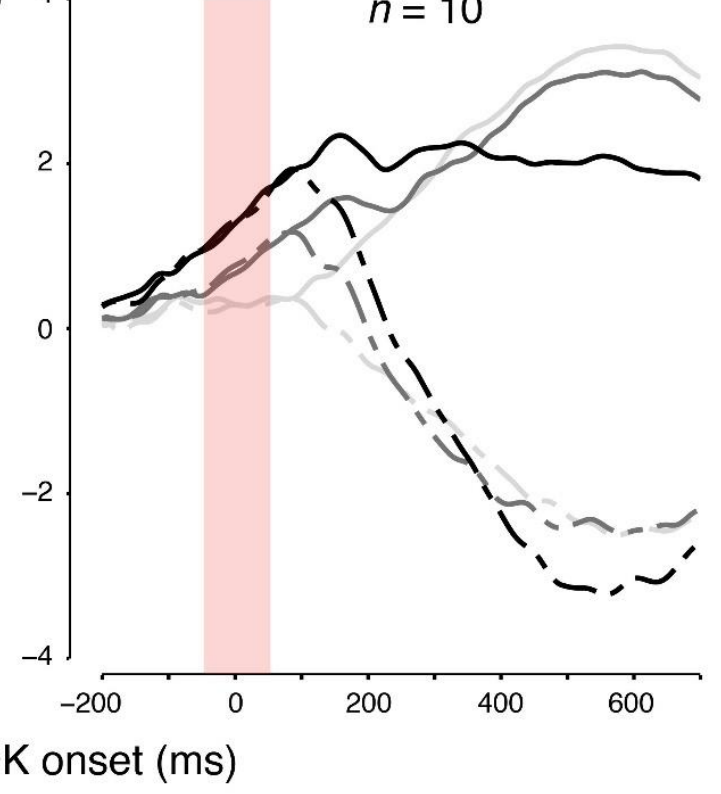

Time from RDK onset (ms)

C

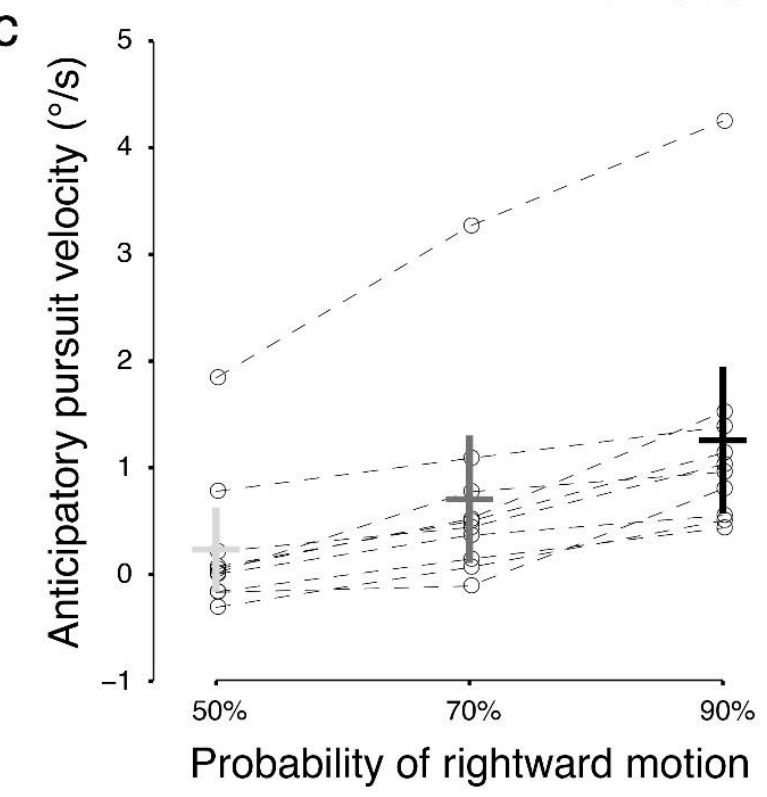

Figure 3. (a) Example trial-average horizontal eye velocity traces in probe trials (leftward or rightward motion direction as indicated by line type) for different probability conditions (indicated by line color) for one representative observer in experiment 1 . The red shaded area indicates the analysis window for anticipatory pursuit. This particular observer had little visuallyguided pursuit in probe trials. (b) Group-average horizontal eye velocity traces in probe trials for different probability conditions in experiment 1 for $n=10$. Line types in panels and $\mathrm{b}$ denote motion direction and probability of rightward motion. (c) Horizontal anticipatory pursuit velocity in experiment 1 averaged across the time interval indicated as shaded area in panel a. Horizontal bars indicate the mean anticipatory pursuit velocity across observers, and vertical bars indicate the $95 \%$ CI. The circles indicate the mean anticipatory pursuit velocity of individual observers, connected by dashed lines across probability conditions. Results were the same even if excluding the one outlier who had relatively high anticipatory pursuit velocity. 
Evidence for repulsion bias in direction perception significant main effect of probability on the PSE (Fig. 4b), $F(2,18)=20.36, p=2.39 \times 10^{-5}, \eta_{g}^{2}$

268 effects of probability on slope $\left(F(2,18)=0.78, p=.48, \eta_{g}^{2}=.02\right)$, indicating that sensitivity did

a

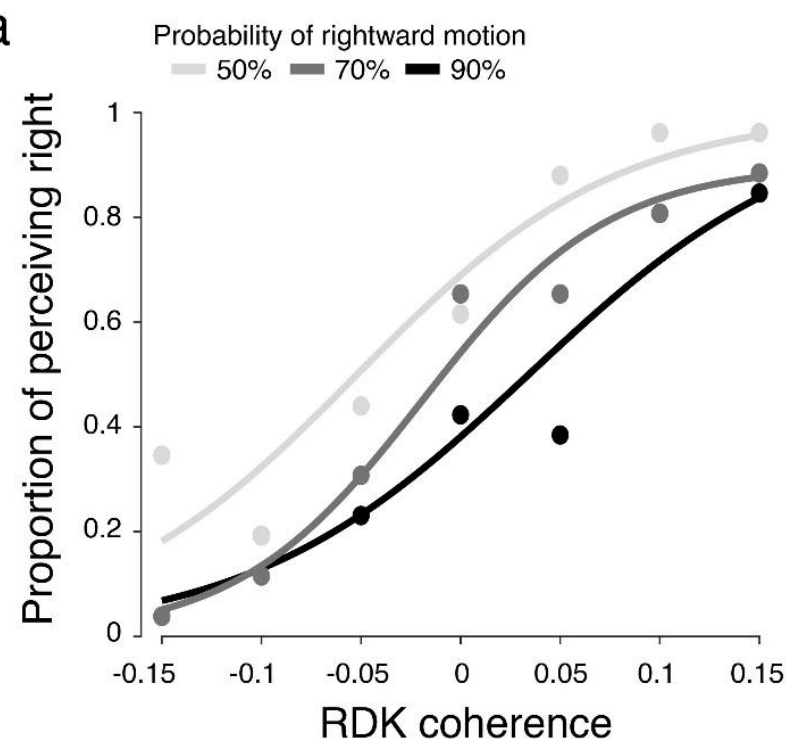

b

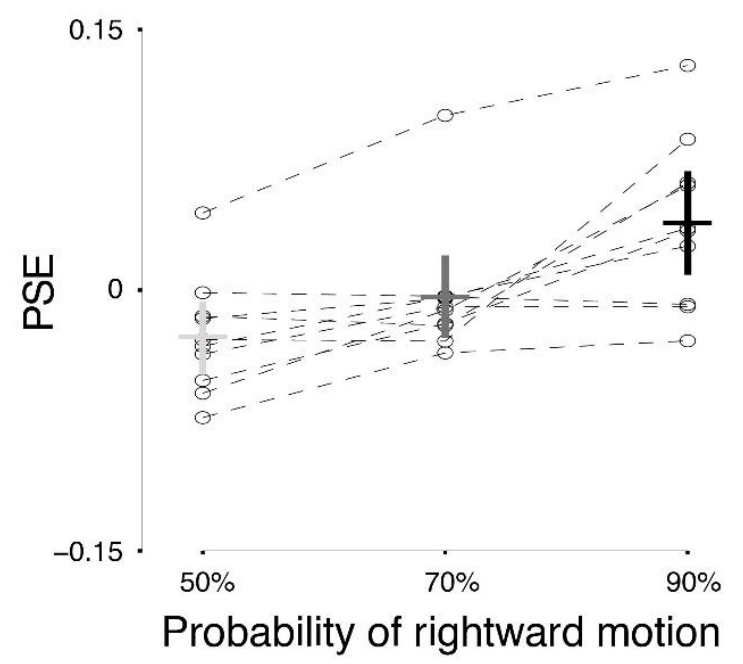

273 Figure 4. (a) Example psychometric curves from the same individual observer shown in Fig. 3a.

274 Negative coherence represents leftward direction. (b) PSE in experiment $1(n=10)$. 
We did not find any dependencies between PSE and anticipatory pursuit velocity in addition to the fact that they both changed with probability. The fixed effect of anticipatory pursuit velocity in the linear mixed-effects model of PSE (PSE anticipatory pursuit velocity + probability $+(1 \mid$ observer $))$ was not significant (estimate \pm std $=-0.002 \pm 0.01, t(20.31)=-0.16$, $p=.87$ ), and only the fixed effect of probability was significant (estimate $\pm s t d=0.002 \pm 0.0004$, $\left.t(30.00)=4.60, p=7.12 \times 10^{-5}\right)$.

Taken together, our results point at a differential effect of motion direction probability on anticipatory pursuit, reflecting an attraction bias, and direction perception, reflecting a repulsion bias. To explore this further, we next examined the potential link between visually-guided pursuit and perception, and analyzed the effect of probability on visually-guided pursuit velocity gain.

\section{Visually-guided pursuit is aligned with direction perception}

Whereas anticipatory pursuit is mostly driven by expectation, visually-guided pursuit is tuned to the visual properties of the target and is known to strongly covary with motion perception (Spering and Montagnini 2011). Previous research has demonstrated that smooth pursuit can be elicited by perceived illusory motion rather than by physical motion (Madelain and Krauzlis 2003; Montagnini et al. 2006). Therefore, visually-guided pursuit could follow different result patterns from anticipatory pursuit and be more aligned with perception.

Here we investigated whether visually-guided pursuit was more in line with an attraction bias (as in anticipatory pursuit) or followed a repulsion bias (as in perception). We compared eye velocity gain in conditions in which the perceptual judgment corresponded to the physical motion direction (congruent) with conditions where perceptual judgments went in the opposite direction to the physical motion (incongruent). Figure 5 shows average velocity traces in probe 
299 trials across all observers for congruent (Fig. 5a) versus incongruent trials (Fig. 5b). Note that

300 this categorization of congruency is agnostic on whether perception followed the expected

301 motion direction or the opposite one and merely reflects how closely perception matched

302 physical target motion. Whereas late visually-guided pursuit followed the visual motion direction

303 in congruent trials (shaded areas in Fig. 5a), pursuit followed visual motion direction less in

304 incongruent trials (Fig. 5b) with a tendency to be directed into the opposite (perceived) direction,

305 resulting in smaller or negative gains. This is confirmed by a significant main effect of

306 congruency on velocity gain $\left(\mathbf{F i g} . \mathbf{5 c} ; F(1,9)=20.65, p=.001, \eta_{p}^{2}=0.70\right)$. The main effect of

307 probability $\left(F(2,18)=0.049, p=.953, \eta_{p}^{2}=.005\right)$ or the congruency $\times$ probability interaction

$308\left(F(2,18)=0.051, p=.950, \eta_{p}^{2}=.006\right)$ were not significant. This difference in visually-guided

309 pursuit between congruent and incongruent conditions persisted across different levels of motion

310 coherence (not shown). 
a

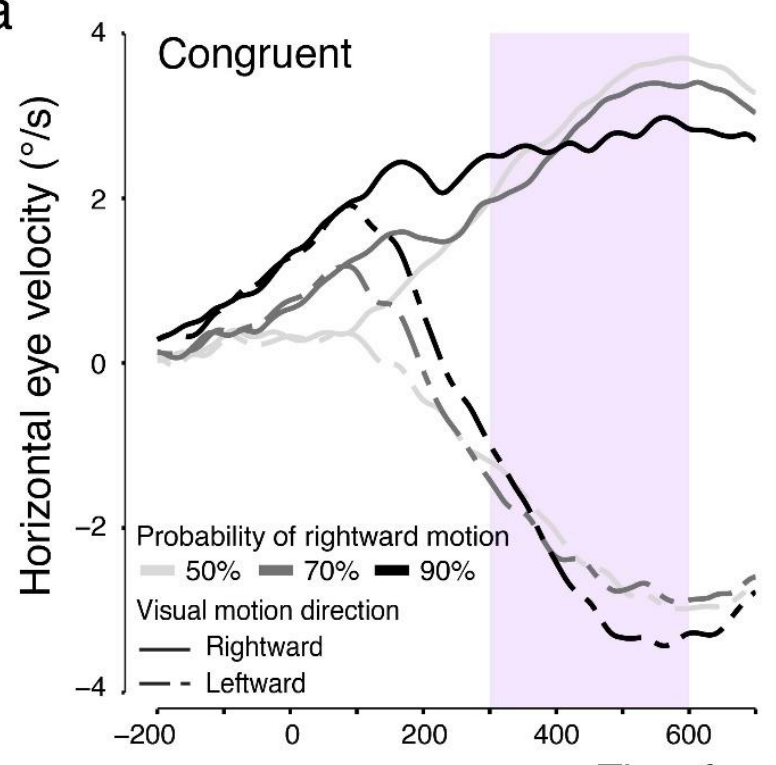

b

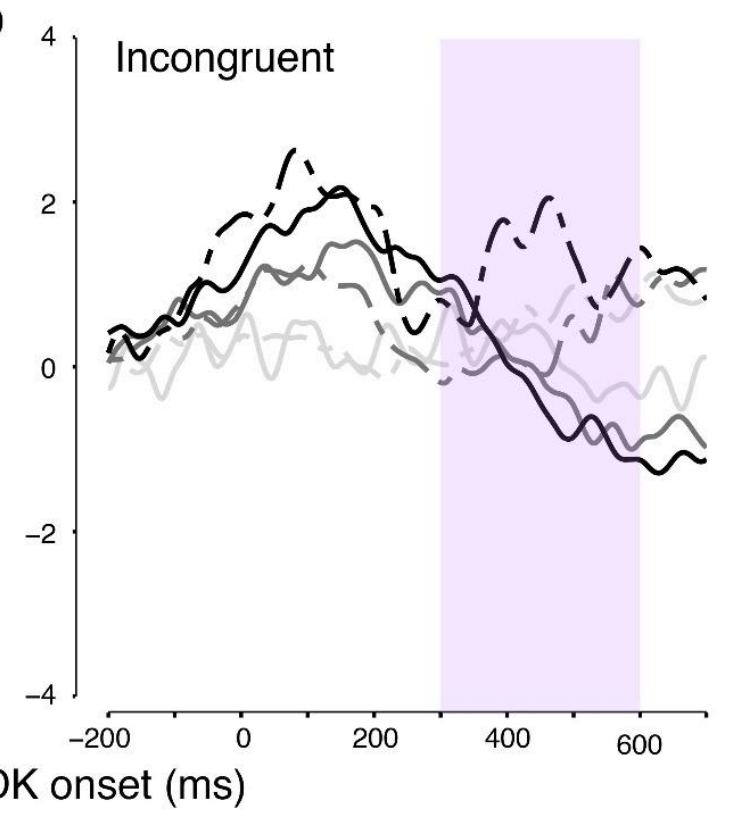

C

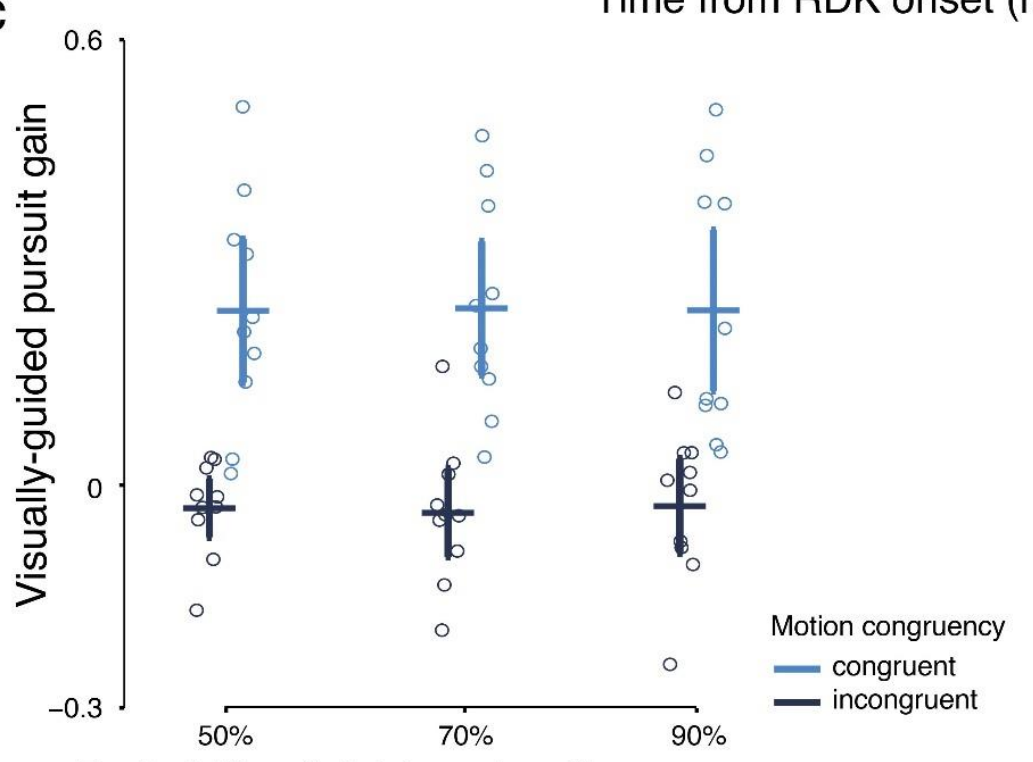

Probability of rightward motion

312 Figure 5. (a) Group-averaged $(n=10)$ horizontal eye velocity traces in probe trials in which 313 visual motion directions were congruent (3967 trials total) with perceived directions across 314 different probability conditions in experiment 1 . The purple shaded area indicates the analysis window for late-phase pursuit; an early cutoff was applied to reduce the effect of anticipatory deceleration before the end of each trial (at $700 \mathrm{~ms})$. (b) Group-averaged $(n=10)$ horizontal eye velocity traces in probe trials in which visual motion directions were incongruent (649 trials total) with perceived directions across different probability conditions in experiment 1. (c) Latephase visually-guided pursuit gain in probe trials grouped by motion congruency across different probability conditions in experiment $1(n=10)$. Higher gain indicates that the eyes follow the visual motion better, and negative gain indicates that the eyes are moving in the opposite direction to the visual motion direction. Horizontal bars indicate the mean visually-guided pursuit gain across observers, and vertical bars indicate the $95 \%$ CI. The circles indicate the 
324 mean visually-guided pursuit gain of individual observers.

Further, we examined if expectation had an effect on visually-guided pursuit, by

327 analyzing the effect of probability on visually-guided pursuit gain. Late-phase visually-guided

328 pursuit in rightward probe trials seemed to have lower velocity in blocks with higher probability

329 of rightward motion (see Fig. 3b, can also be seen in Fig. 5a with majority of probe trials). Since

330 direction expectation might affect visually-guided smooth pursuit differently for rightward and

331 leftward motion trials, we included visual motion direction as a second factor in the two-way

332 ANOVA on visually-guided pursuit gain. If the observed decrease in eye velocity with increased

333 probability across blocks was true, a significant main effect of probability and possibly a

334 significant interaction effect of probability $\times$ visual motion direction could be observed.

335 However, the interaction effect $\left(F(2,18)=1.54, p=.24, \eta_{p}^{2}=.15\right.$; Fig. 6), the main effect of

336 probability $\left(F(2,18)=0.58, p=.57, \eta_{p}^{2}=.06\right)$, or the main effect of visual motion direction

$337\left(F(1,9)=1.87, p=.20, \eta_{p}^{2}=.17\right)$ were non-significant. This could be due to the large individual

338 variability - some observers had little visually-guided pursuit in probe trials across probability

339 conditions (gain close to zero), likely due to the low RDK coherence. 


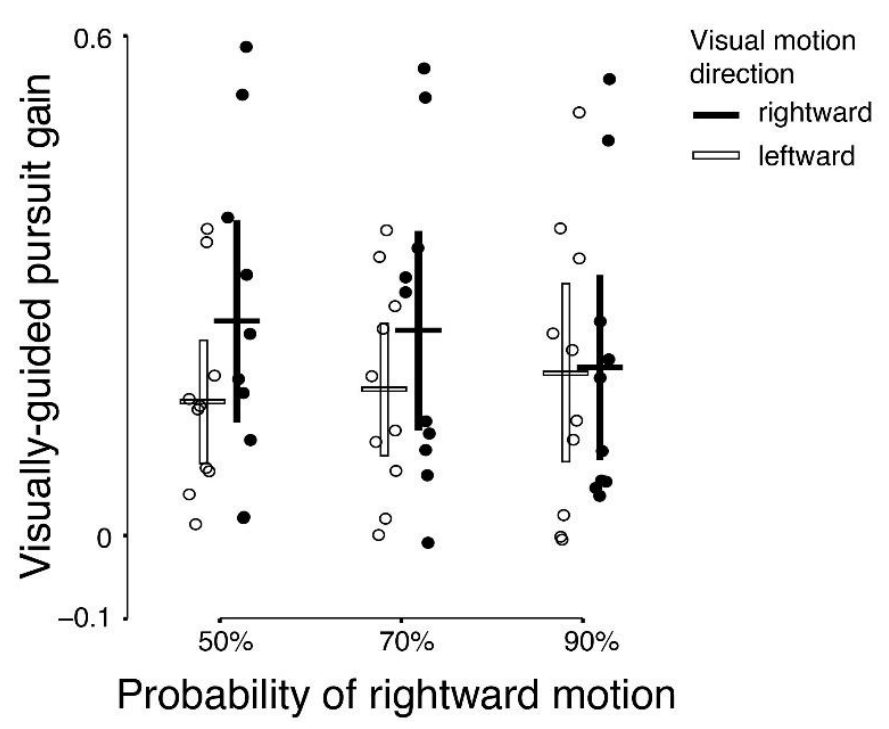

Figure 6. Late-phase visually-guided pursuit gain in probe trials grouped by visual motion direction across different probability conditions in experiment $1(n=10)$.

Notwithstanding a lack of evidence for expectation effects on visually-guided pursuit,

347 finding indicates that late-phase pursuit is driven by signals that are more coherent with those 348 signals driving perceptual judgments than those driving anticipatory pursuit.

\section{Experiment 2}

To further explore the mechanisms underlying the dissociation between expectation

352 effects on anticipatory pursuit and perception, we conducted two control experiments. One

353 potential problem with our paradigm might be that anticipatory pursuit during the earliest phase

354 of the presentation of the low-coherence RDK elicits retinal image motion in the opposite

355 direction than the expected one. This motion signal could have informed the perceptual choice,

356 explaining the repulsion bias. In experiment 2, we therefore tested whether the observed

357 perceptual bias was affected by this negative retinal motion signal by manipulating anticipatory

358 pursuit magnitude. To reduce anticipatory pursuit, we showed the fixation point until RDK onset, 
omitting the 300-ms gap introduced in Experiment 1, and instructed observers to maintain

360 fixation until the stimulus started moving. Each observer completed two blocks (50\% and $90 \%$

361 probability of rightward motion). All other procedures were the same as in experiment 1.

362 To confirm that anticipatory pursuit was reduced in Experiment 2 as compared to

363 Experiment 1, we performed a two-way repeated measures ANOVA with experiment and

364 probability as factors. An experiment $\times$ probability interaction effect on anticipatory pursuit

365 velocity would indicate a change in anticipatory pursuit magnitude from one experiment to the

366 other. If anticipatory pursuit induced the perceptual bias, reduced anticipatory pursuit magnitude

367 in Experiment 2 should result in a smaller perceptual bias. This interpretation would be

368 supported by a significant experiment $\times$ probability interaction effect on PSE.

Anticipatory pursuit was significantly reduced with prolonged fixation

The experimental manipulation of prolonging fixation in Experiment 2 yielded the expected reduction in anticipatory pursuit velocity from $1.26 \pm 1.11 \% \mathrm{~s}(M \pm s t d)$ in Experiment 1 to $0.57 \pm 0.30 \%$ in Experiment 2 at the highest probability of rightward motion (Fig. 7a, b).

374 This observation was confirmed by a significant experiment $\times$ probability interaction (Fig. 7c;

$\left.375 F(1,7)=7.20, p=.03, \eta_{p}^{2}=.51\right)$. Despite lower overall velocity, higher probability of rightward

376 motion continued to induce higher anticipatory pursuit velocity, reflected in a main effect of

377 probability $\left(F(1,7)=37.81, p=.0005, \eta_{p}^{2}=.84\right)$. Congruently, the bootstrapped mean and $95 \%$

$378 \mathrm{CI}$ of anticipatory pursuit velocity in experiment 2 were $.05 \pm .01 \% \mathrm{~s}$ for $50 \%$ and $.59 \pm .01 \% \mathrm{~s}$ for

$37990 \%$. The main effect of experiment was not significant $\left(F(1,7)=2.06, p=.19, \eta_{p}^{2}=.23\right)$. 
a

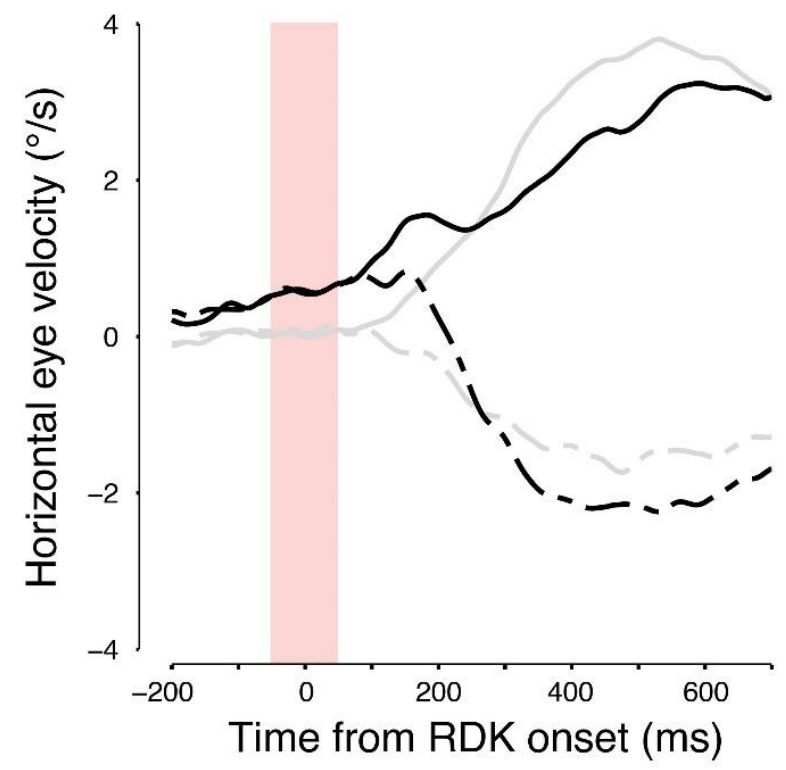

b

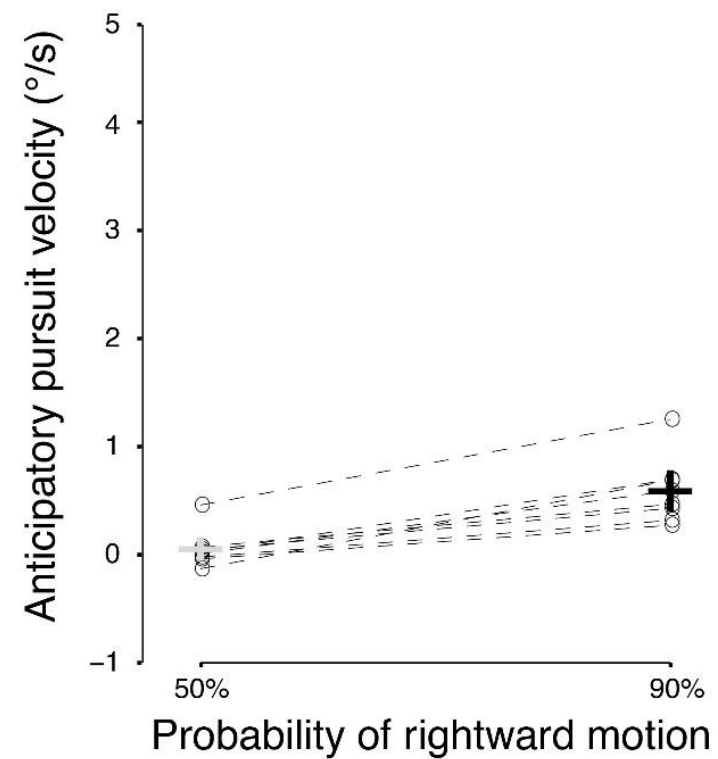

Probability of rightward motion

$=50 \%-90 \%$

Visual motion direction

— Rightward

- - Leftward

Figure 7. (a) The group-averaged $(n=8)$ horizontal eye velocity traces in probe trials across different probability conditions in experiment 2. (b) Horizontal anticipatory pursuit velocity in experiment $2(n=8)$. (c) The comparison of anticipatory pursuit velocity between experiment 1 and 2. The horizontal bars show the mean across observers ( $n=8$ for both experiments), and the error bars show the $95 \%$ CI.

Persistent perceptual bias despite reduced anticipatory pursuit velocity

Despite the successful reduction in anticipatory pursuit velocity, we observed the same repulsion bias (rightward shift of the PSE; Fig. 8a) in perceptual judgments in Experiment 2. 
391 This observation was confirmed by a significant main effect of probability, $F(1,7)=22.91, p$

$392=.002, \eta_{p}^{2}=.77$, and a lack of experiment $\times$ probability interaction $($ Fig. $\mathbf{8 b} ; F(1,7)=0.001, p$

$\left.393=.97, \eta_{p}^{2}=.0002\right)$. The bootstrapped mean and 95\% CI of the PSE were $-.04 \pm .003$ for $50 \%$

394 and $.02 \pm .002$ for $90 \%$. The main effect of experiment was not significant $(F(1,7)=4.54, p$

$\left.395=.07, \eta_{p}^{2}=.39\right)$. These results indicate that the perceptual bias remained stable across

396 experiments, and that negative retinal image motion induced by anticipatory pursuit is unlikely to

397 cause the repulsion bias.

398

a

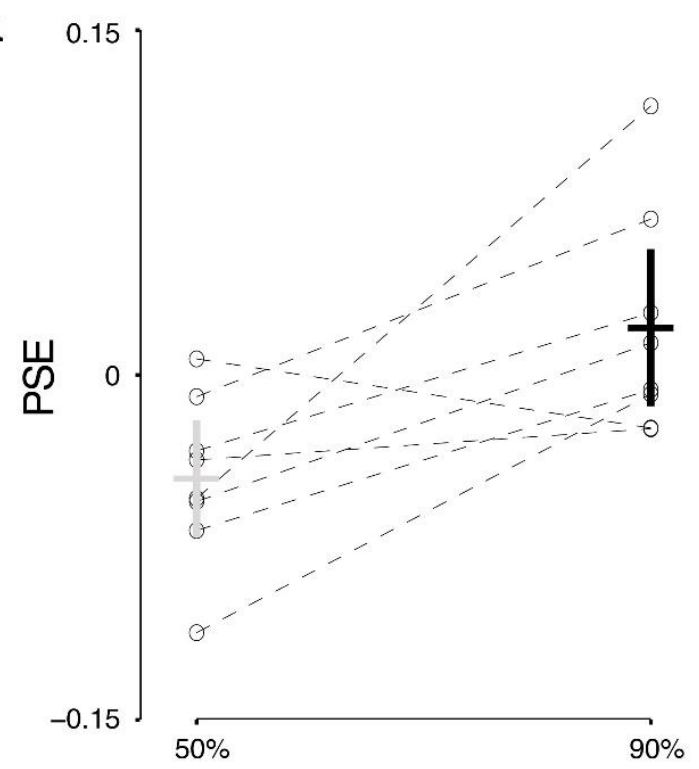

Probability of rightward motion

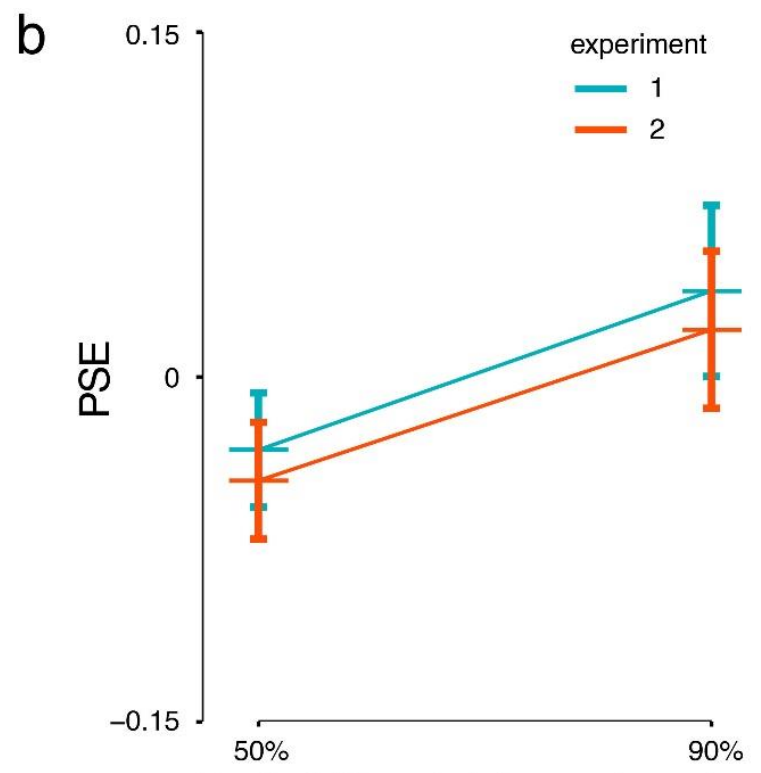

Probability of rightward motion

Figure 8. (a) PSE in probe trials across different probability conditions in experiment $2(n=8)$. (b) The comparison of PSE between experiment 1 and 2 ( $n=8$ in each experiment).

\section{Experiment 3}

In all experiments presented so far, we used a noise mask following RDK presentation 
perceptual repulsion effect observed in experiments 1 and 2 could be that prolonged exposure to

409 a high-coherence moving stimulus in context trials produces a perceptual aftereffect (a form of

410 low-level sensory adaptation) despite the mask. One way to reduce potential effects of motion

411 aftereffects or other similar forms of sensory adaption is to lower the motion signal strength of

412 the adaptor, for example, by reducing its luminance contrast (Keck et al. 1976). It is well known

413 that the response of neurons in motion-sensitive middle temporal cortex (area MT) is modulated

414 by motion coherence (Händel et al. 2007). In Experiment 3, we therefore reduced the coherence

415 of the RDK in context trials to investigate whether such a manipulation would weaken the

416 perceptual repulsion bias. We reduced motion coherence of RDKs in context trials to $25 \%$ on

417 average (coherence levels of $20 \%$ and $30 \%$ randomly assigned to half of the context trials in each

418 block). This coherence level is considered to be above perceptual thresholds for direction

419 discrimination in adults (Meier and Giaschi 2014) and yielded judgements of $>99 \%$ correct

420 accuracy in context trials in our experiment. We therefore expected that the perceived probability

421 of context trials (50\% and 90\%) remained the same as in previous experiments. All other

422 procedures were the same as in experiment 1.

423 First, we assessed whether coherence impacted visually-guided pursuit in context trials to

424 confirm that the coherence manipulation successfully reduced the motion signal. We conducted a

425 two-way repeated-measures ANOVA on pursuit gain with experiment and probability as factors.

426 A significant main effect of experiment would imply a reduction in motion signal due to the

427 reduced coherence. To examine if motion coherence has an effect on anticipatory pursuit, we

428 conducted a two-way repeated-measures ANOVA on anticipatory pursuit velocity with

429 experiment and probability as factors. A significant interaction would indicate that anticipatory

430 pursuit was modulated by motion signal strength in context trials. Second, to examine whether 
431 RDK coherence in context trials affects perception, we conducted a two-way repeated-measures

432 ANOVA on PSE with experiment and probability as factors. If RDK coherence in context trials

433 affected the repulsion bias, we should find a significant interaction.

The experimental manipulation of motion coherence yielded the expected reduction in visually-guided pursuit gain in context trials (exp. 1: $M=0.89 \pm 0.11$ across observers and probability conditions, exp. 3: $M=0.49 \pm 0.16)$. This observation was confirmed by a significant main effect of experiment on pursuit gain $\left(F(1,8)=192.62, p=7.03 \times 10^{-7}, \eta_{p}^{2}=.96\right)$. No significant main effect of probability $\left(F(1,8)=1.66, p=.23, \eta_{p}^{2}=.17\right)$ or interaction $(F(1,8)=$ a weaker motion signal and therefore lower pursuit gain in context trials.

444 anticipatory pursuit velocity at the highest probability of rightward motion in probe trials (exp. 1:

$445 M=1.26 \pm 1.11 \%$ s; exp. 3: $M=0.62 \pm 0.62 \%$, Fig. 9a, b). This observation was confirmed by a

446 significant experiment $\times$ probability interaction effect $\left(F(1,8)=32.39, p=.0005, \eta_{p}^{2}=0.80\right)$,

447 indicating that the effect of probability was smaller in experiment 3 than in experiment 1 (Fig. 3c).

448 The main effect of probability was also significant $\left(F(1,8)=23.29, p=.001, \eta_{p}^{2}=.74\right)$, and the

449 main effect of experiment was not significant $\left(F(1,8)=5.10, p=.05, \eta_{p}^{2}=.39\right)$. The bootstrapped

450 mean and $95 \%$ CI of anticipatory pursuit velocity in experiment 3 were $0.17 \pm 0.01 \%$ for $50 \%$ and 
a

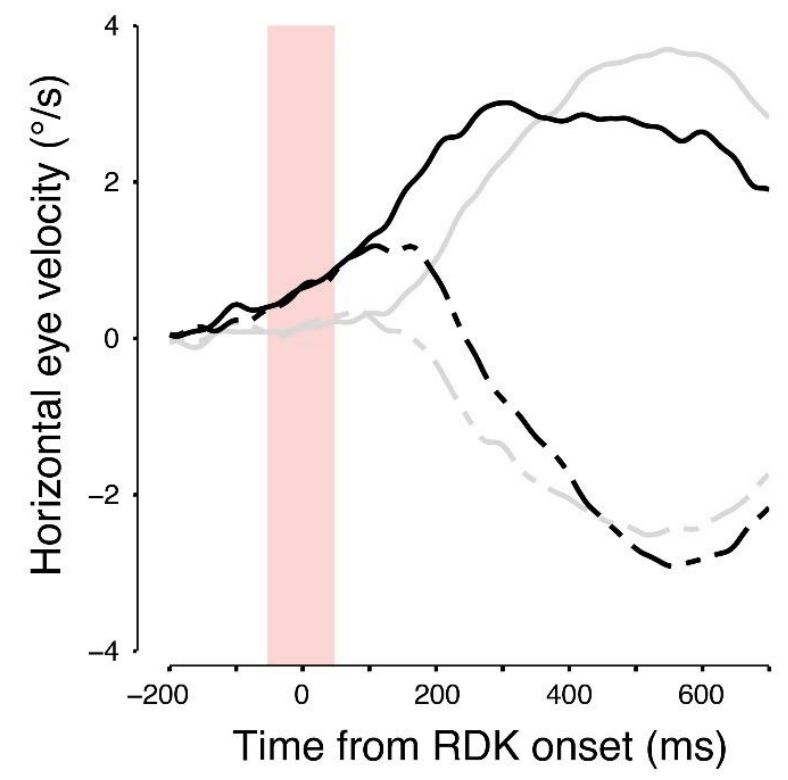

b

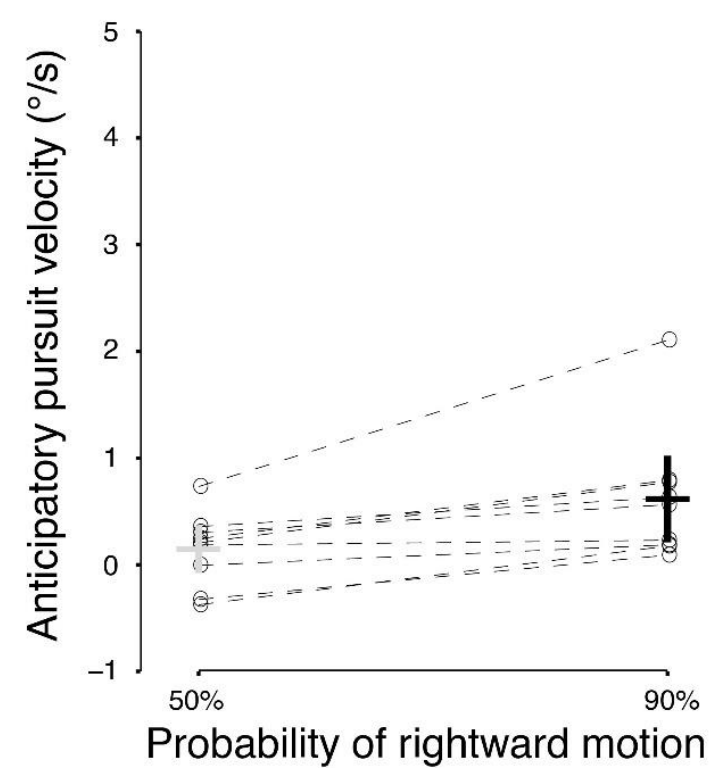

Probability of rightward motion

$-50 \%=90 \%$

Visual motion direction

— Rightward

- - Leftward

C



$453 \quad$ Figure 9. (a) The averaged $(n=9)$ horizontal eye velocity traces in probe trials across different 454 probability conditions in experiment 3. (b) Horizontal anticipatory pursuit velocity in 455 experiment $3(n=9)$. (c) The comparison of anticipatory pursuit velocity between experiment 1 456 and $3(n=9$ in each experiment). 
to cause sensory adaptation, the perceptual bias remained stable (Fig. 10). This observation was

$\left.463 \eta_{p}^{2}=.06\right)$, indicating a similar magnitude of perceptual bias in both experiments (Fig. 10). The

464 main effect of probability was significant $\left(F(1,8)=44.97, p=.0002, \eta_{p}^{2}=.85\right)$, and the main

465 effect of experiment was not significant $\left(F(1,8)=0.13, p=.73, \eta_{p}^{2}=.02\right)$. Congruently, the

466 bootstrapped mean and 95\% CI of the PSE were $-.02 \pm .002$ for $50 \%$ and $.05 \pm .002$ for $90 \%$.

a



b

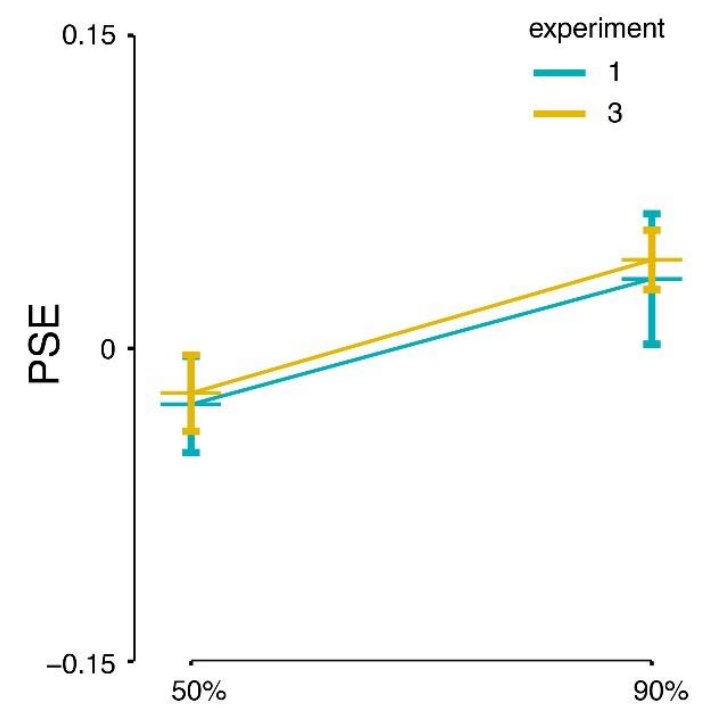

Probability of rightward motion

Figure 10. (a) PSE in probe trials across different probability conditions in experiment $3(n=9)$. (b) Comparison of PSE between experiment 1 and 3 ( $n=9$ in each experiment).

Discussion 
477 expectation affected anticipatory pursuit and direction perception. Anticipatory pursuit was

478 directed toward the highest probability direction in a given block of trials. By contrast, the direction

479 of perceptual judgments was repelled away from the most probable direction. This repulsion bias

480 was not caused by anticipatory pursuit (experiment 2) and unlikely to be caused by sensory

481 adaptation (experiment 3 ).

482 These results make a novel contribution to the literature on the comparison between

483 perception and pursuit and highlight that motion perception does not necessarily rely on

484 expectation in the same way as early, anticipatory pursuit responses do. These findings are

485 generally congruent with a previous study investigating trial history effects in the velocity

486 domain (Maus et al. 2015). In that study, the authors presented random sequences of brief, dot-

487 motion stimuli with different speeds. Observers were asked to track the motion with their eyes

488 and judge whether the current trial's speed was faster or slower than the speed averaged across

489 previous trials. Whereas anticipatory pursuit scaled with previous target speed, perceptual

490 judgments were faster after slow stimuli and vice versa for fast stimuli, indicating a similar

491 repulsion effect as observed for motion direction in the current study. Taken together, our study

492 and the previous study show that opposite effects on perception and anticipatory pursuit exist

493 regardless of which feature of the stimulus is manipulated (speed vs. direction) and over which

494 time course both responses are compared (long-term prior vs. shorter-term trial history).

496 in perception and pursuit are generally compatible with the idea of different information

497 processing for both responses. Perception and visually-guided pursuit eye movements might rely

498 on different information accumulation and integration over time, due to different needs of the

499 perceptual and the oculomotor systems (Spering and Montagnini 2011). The current paper 
mainly compares perceptual responses to another aspect of pursuit, the earliest, anticipatory

501 phase that is driven by cognitive, memory-related signals rather than by visual signals.

502 Interestingly, the present study did not find a significant effect of direction expectation on the

503 later, visually-guided phase of smooth pursuit. However, in trials in which the perceived

504 direction was incongruent with the physical motion direction (i.e., followed a repulsion bias),

505 visually-guided smooth pursuit was aligned with perception. In the following paragraphs, we will

506 discuss the characteristics of the signals driving motion perception and different phases of

507 smooth pursuit with a main focus on anticipatory pursuit.

Different biases in motion perception and pursuit might reflect how both responses adapt to task

510 requirements

511 Studies on perceptual responses to manipulations in short-term or long-term probability

512 have mostly revealed an attraction bias (Alais et al. 2017; Chalk et al. 2010; Kok et al. 2013), in

513 which perception follows the recently viewed or most likely stimulus feature (direction,

514 orientation, etc.). Our paradigm utilized short-duration displays and introduced a statistical bias

515 of motion direction (long-term probability), similar to some of these studies (e.g., Chalk et al.

516 2010). However, we observed a repulsion bias. These different types of biases are interesting

517 because they reveal that the perceptual system might respond to different task and stimulus

518 environments in a flexible way, depending on the requirements of the task (e.g., to sensitively

519 respond to a change or categorize information). A repulsion bias might reflect the need of the

520 perceptual system to stay alert and to quickly respond to changes in the environment in an

521 energy-efficient way: there is no need to be highly sensitive to a stimulus that always appears,

522 whereas a novel stimulus would alert the system and may require priority processing. This is 
523 similar to the functional role of adaptation, yet results from experiment 3 suggested that the

524 repulsion perceptual bias in our experiment was not caused by low-level sensory adaptation.

525 Similarly, different result patterns in perception and pursuit might reflect different task

526 requirements as well. Our results resemble those obtained in other studies comparing perception

527 and pursuit (Spering and Gegenfurtner 2007), or pursuit and hand movements (Kreyenmeier et

528 al. 2017). These studies found that pursuit generally followed the motion average of different

529 target and context speeds, whereas perception and manual interception of a target followed the

530 difference of target and background. Akin to the task requirements in our study, perception and

531 action served different functions in these studies as well. Whereas perception's role appeared to

532 be to segregate a target from the background, pursuit's role was to track in the general target

533 direction in the presence of surrounding motion signals, resulting in motion vector averaging. In

534 the present work the tendency for pursuit to rely on integration rather than segmentation of

535 different signals seems to be extended to the trial-history domain, and to the anticipatory phase:

536 integration of visual signals across many trials would drive anticipatory pursuit, whereas

537 perception could be based on the contrast between the current visual input and long-term

538 memory of visual motion.

540 Expectation effects on anticipatory vs. visually-guided pursuit

Anticipatory pursuit has been shown to be not purely habitual (Jarrett and Barnes 2001,

542 2002; Kowler 1989), but also sensitive to different types of cognitive cues and probabilistic

543 context (Pasturel et al. 2020; Santos and Kowler 2017) as well as to reward (Damasse et al.

544 2018). In addition, we show that anticipatory pursuit velocity is modulated by motion coherence

545 in context trials, i.e. motion signal strength in the prior history. Our results together with previous 
546 findings suggest that anticipatory pursuit is based on the integration of multiple signals, from

547 low-level visual motion signals (weighted by the sensory strength, or saliency, e.g. RDK

548 coherence), to higher-level cognitive cues such as expectation and reward. This holds across the

549 short time-scale of a single trial for visually-guided early smooth pursuit (e.g., Ferrera and

550 Lisberger 1997) and a much longer time-scale lasting several minutes for anticipatory pursuit, as

551 in our experimental blocks. The assumption that anticipatory pursuit is based on such an

552 integration of multiple signals is reasonable considering that the goal of anticipatory pursuit is to

553 reach accurate tracking as soon as possible, in order to reduce the temporal delay in tracking the

554 visual target.

555 Interestingly, whereas anticipatory pursuit showed an attraction bias scaling with

556 probability, late-phase visually-guided pursuit did not follow the same result pattern. This

557 difference might be expected given that visually-guided pursuit and perception similarly rely on

558 current sensory signals, whereas anticipatory pursuit is driven by expectation. Moreover, it is

559 known that visually-guided pursuit and motion perception interact, and that motion perception

560 can modulate pursuit (Madelain and Krauzlis 2003; Montagnini et al. 2006). It is thus possible

561 that the late-phase visually-guided pursuit observed in our experiment was driven by perception,

562 regardless of the nature of the expectation information.

Neural correlates of expectation effects on perceptual bias and anticipatory pursuit

The dissociation between effects of expectation on perception and anticipatory pursuit

566 might be due to perception and pursuit depending on different cortical areas during different

567 processing stages. For perception, modulation by expectation might have affected sensory

568 processing of current stimuli, based on activity in early visual cortical areas. By contrast, 
anticipatory pursuit is not triggered by current stimuli but instead based on expectation or history, related to activity in frontal cortical areas. In the following paragraphs we will discuss

571 the neural correlates for perception and anticipatory pursuit accordingly.

574 Bradley 2005; Thier and Ilg 2005). It is unclear which specific cortical areas are responsible for expectation effects on perception. However, there is evidence that modulation in early sensory cortices, from primary visual cortex (V1) to MT and MST, might underlie repulsion and

577 attraction biases in perception. For example, the repulsion bias in perceived orientation was

578 found to be stronger when the current and previous stimuli in a given trial were presented at the 579 same location (Fritsche et al. 2020). These findings indicate that this orientation bias was driven by effects that are spatially specific (and retinotopically congruent), which likely implies

581 modulation of neurons in early sensory cortex, such as V1, responsive to stimuli within small

582 receptive fields. Similarly, spatial specificity has been found in visual motion adaptation in

583 relation to neuronal activity in area MT (Kohn and Movshon 2003). Area MT is known for its

584 large receptive fields and might inherit spatial specificity from V1, but also shows distinct

585 adaptation responses (Kohn and Movshon 2004) that could underlie a repulsion bias. Although

586 our results indicate that such low-level sensory adaptation was unlikely the cause of the repulsion

587 bias in our study, other mechanisms could have resulted in a similar modulation of MT neuronal

588 activity, leading to a repulsion bias in perception. Congruently, for attraction biases induced by

589 expectation, functional magnetic resonance imaging studies (e.g., Kok et al. 2013) showed that

590 representation of visual motion direction in early sensory cortices from V1 to MT was biased

591 toward the expected stimulus. Taken together, these studies indicate that sensory areas as early as 
$592 \mathrm{~V} 1$, and potentially up to MT and MST, play an important role in the effect of expectation on

593 motion perception. Whether the modulation on early sensory cortex comes from higher-level

594 areas remains unclear.

595 The supplementary eye field (SEF) plays a critical role for anticipatory pursuit, as shown

596 in studies using electrical microstimulation in SEF to elicit anticipatory pursuit (Missal and

597 Heinen 2004). Moreover, direction-selective neurons in area SEF showed stronger activity before

598 anticipatory pursuit in their preferred direction, indicating that SEF plays a role in the

599 preparation of anticipatory pursuit (de Hemptinne et al. 2008). The pursuit area of the frontal eye

600 field $\left(\mathrm{FEF}_{\text {sem }}\right)$ could also contribute to anticipatory pursuit, because lesions in FEF could abolish

601 the ipsilateral anticipatory initiation of pursuit (Macavoy et al. 1991). We hypothesize that

602 expectation based on visual and/or motor history might be encoded in SEF and then combined

603 with current sensory evidence in FEF (Darlington et al. 2018; Fukushima et al. 2013; Schall

604 2015). The source of visual motion history might come from MT and MST, but the roles of these

605 areas in anticipatory pursuit remain to be tested (Kowler et al. 2019).

\section{Limitations and future directions}

608 One limitation of the current study is that we were not able to analyze the temporal

609 development of the expectation effect in perception in detail due to limited number of probe

610 trials. Anticipatory pursuit and perception rely on history on different temporal scales (Maus et

611 al. 2015). Attraction and repulsion perceptual bias seem to also operate on different time scales

612 (Chopin and Mamassian 2012; Fritsche et al. 2020), and could occur with slight changes of

613 parameters in the same paradigm (Kanai and Verstraten 2005). Therefore, understanding the

614 temporal dependency and development of the effects of expectation would be crucial to 
615 understand the complicated interaction between attraction and repulsion biases.

616 We do not know how robust our results are with regard to parametric variations of the

617 visual stimuli. For example, an attraction bias is mostly observed in studies using moving stimuli

618 whose directions differed by about $60^{\circ}$ or less (Fritsche et al. 2020) rather than $180^{\circ}$ (as in our

619 study). In addition, effects of expectation are often examined with weak motion stimuli, i.e., low

620 contrast (Chalk et al. 2010) or low coherence (probe trials in our study), because the Bayesian

621 integration hypothesis postulates that the effect of expectation would be larger on a stimulus with

622 less reliable sensory signals. However, reducing coherence might introduce changes other than

623 reducing contrast for RDK stimuli, such as inducing the perceptual phenomenon of motion

624 transparency, in which two or more distinct surfaces are perceived as moving in different

625 direction (Qian et al. 1994). Motion characteristics of noise dots in an RDK, together with their

626 lifetime, affect the perception of global motion as well as pursuit quality (Pilly and Seitz 2009;

627 Schütz et al. 2010). Future work is needed to elucidate the potential influence of the

628 characteristics of sensory stimuli — ranging from simplified dots, blobs and RDKs to more

629 complex naturalistic stimuli (Goettker et al. 2020)—on behavioral biases in perception and eye

630 movements.

631 Finally, the perceptual repulsion bias observed in our and other studies does not match

632 predictions of optimal Bayesian integration. Standard Bayesian inference would predict an

633 attraction bias to the prior. Combining this prediction with the efficient coding hypothesis,

634 whereby expectation modulates sensory likelihood, could account for the repulsion biases

635 (“Anti-Bayesian” effects; Wei and Stocker 2015). In the future, this kind of modeling approach

636 might help understand the complicated interaction between attraction and repulsion biases

637 induced by experience-based expectation across different behavioral tasks. 


\section{Acknowledgements}

This work was supported by a University of British Columbia four-year fellowship to

641 X.W., a Natural Sciences and Engineering Research Council of Canada Discovery Grant and

642 Accelerator Supplement to M.S., and a CNRS - PICS grant "APPVIS" to A.M. Preliminary data

643 from this project were presented at the 2019 Gordon Research Conference on Eye Movements,

644 the 2019 Gordon Research Seminar on Eye Movements, the Society for Neuroscience annual

645 meeting (Wu et al. 2019), and the Vision Sciences Society annual meeting (Wu et al. 2020). The

646 authors thank members of the Spering lab for comments on an earlier draft of the article.

$648 \quad$ References

649 Alais D, Leung J, Van der Burg E. Linear summation of repulsive and attractive serial 650 dependencies: Orientation and motion dependencies sum in motion perception. $J$ Neurosci 37:

$651 \quad 4381-4390,2017$.

652 Bates D, Mächler M, Bolker BM, Walker SC. Fitting linear mixed-effects models using lme4. J

653 Stat Softw 67: 1-48, 2015.

654 Behling S, Lisberger SG. Different mechanisms for modulation of the initiation and steady-state 655 of smooth pursuit eye movements. J Neurophysiol 123: 1265-1276, 2020.

656 Born RT, Bradley DC. Structure and function of visual area MT. Annu Rev Neurosci 28: 157$657189,2005$.

658 Brainard DH. The Psychophysics Toolbox. Spat Vis 10: 433-6, 1997.

659 Braun DI, Pracejus L, Gegenfurtner KR. Motion aftereffect elicits smooth pursuit eye 660 movements. J Vis 6: 671-684, 2006. 
Chalk M, Seitz AR, Series P. Rapidly learned stimulus expectations alter perception of motion.

JVis 10: 1-17, 2010.

663 Chopin A, Mamassian P. Predictive Properties of Visual Adaptation. Curr Biol 22: 622-626, 6642012.

665 Cicchini GM, Mikellidou K, Burr DC. The functional role of serial dependence. Proc $R$ Soc B 666 Biol Sci 285: 1-8, 2018.

667 Damasse J-B, Perrinet LU, Madelain L, Montagnini A. Reinforcement effects in anticipatory 668 smooth eye movements. J Vis 18: 1-18, 2018.

669 Darlington TR, Beck JM, Lisberger SG. Neural implementation of Bayesian inference in a 670 sensorimotor behavior. Nat Neurosci 21: 1442-1451, 2018.

671 Darlington TR, Tokiyama S, Lisberger SG. Control of the strength of visual-motor transmission 672 as the mechanism of rapid adaptation of priors for Bayesian inference in smooth pursuit eye 673 movements. J Neurophysiol 118: 1173-1189, 2017.

674 Deravet N, Blohm G, de Xivry J-JO, Lefèvre P. Weighted integration of short-term memory and 675 sensory signals in the oculomotor system. J Vis 18: 1-19, 2018.

676 Ferrera VP, Lisberger SG. Neuronal Responses in Visual Areas MT and MST During Smooth 677 Pursuit Target Selection. J Neurophysiol 78: 1433-1446, 1997.

678 Fritsche M, Spaak E, de Lange FP. A Bayesian and efficient observer model explains concurrent 679 attractive and repulsive history biases in visual perception. Elife 9, 2020.

680 Fukushima K, Fukushima J, Warabi T, Barnes GR. Cognitive processes involved in smooth 681 pursuit eye movements: Behavioral evidence, neural substrate and clinical correlation. Front. Syst. 682 Neurosci. 7Frontiers: 1-28, 2013.

683 Gegenfurtner KR. The Interaction Between Vision and Eye Movements. Perception 45: 1333- 
1357, 2016.

685 Goettker A, Agtzidis I, Braun DI, Dorr M, Gegenfurtner KR. From Gaussian blobs to 686 naturalistic videos: Comparison of oculomotor behavior across different stimulus complexities. $J$

687 Vis 20: 1-16, 2020.

689 Coherence in Human Area MT1 and Early Visual Cortex. Cereb Cortex 17: 1542-1549, 2007.

690 de Hemptinne C, Lefèvre P, Missal M. Neuronal bases of directional expectation and 691 anticipatory pursuit. J Neurosci 28: 4298-4310, 2008.

692 Jarrett CB, Barnes G. Volitional selection of direction in the generation of anticipatory ocular 693 smooth pursuit in humans. Neurosci Lett 312: 25-28, 2001.

694 Jarrett CB, Barnes G. Volitional scaling of anticipatory ocular pursuit velocity using precues. 695 Cogn Brain Res 14: 383-388, 2002.

696 Jazayeri M, Movshon JA. A new perceptual illusion reveals mechanisms of sensory decoding. 697 Nature 446: 912-915, 2007.

698 Kanai R, Verstraten FAJ. Perceptual manifestations of fast neural plasticity: Motion priming, 699 rapid motion aftereffect and perceptual sensitization. Vision Res 45: 3109-3116, 2005.

700 Keck MJ, Palella TD, Pantle A. Motion aftereffect as a function of the contrast of sinusoidal 701 gratings. Vision Res 16: 187-191, 1976.

702 Kleiner M, Brainard D, Pelli D., Ingling A, Murray R, Broussard C. What's new in 703 psychtoolbox-3. Perception 36: 1-16, 2007.

704 Kohn A, Movshon JA. Neuronal adaptation to visual motion in area MT of the macaque. Neuron 705 39: 681-691, 2003.

706 Kohn A, Movshon JA. Adaptation changes the direction tuning of macaque MT neurons. Nat 
Neurosci 7: 764-772, 2004.

708 Kok P, Brouwer GJ, van Gerven MAJ, de Lange FP. Prior expectations bias sensory

709 representations in visual cortex. J Neurosci 33: 16275-16284, 2013.

710 Kowler E. Cognitive expectations, not habits, control anticipatory smooth oculomotor pursuit.

711 Vision Res 29: 1049-1057, 1989.

712 Kowler E, Martins AJ, Pavel M. The effect of expectations on slow oculomotor control-IV.

713 Anticipatory smooth eye movements depend on prior target motions. Vision Res 24: 197-210, 1984.

714 Kowler E, Rubinstein JF, Santos EM, Wang J. Predictive Smooth Pursuit Eye Movements.

715 Апnu Rev Vis Sci 5: 223-246, 2019.

716 Krauzlis RJ, Miles FA. Release of fixation for pursuit and saccades in humans: Evidence for

717 shared inputs acting on different neural substrates. J Neurophysiol 76: 2822-2833, 1996.

718 Kreyenmeier P, Fooken J, Spering M. Context effects on smooth pursuit and manual

719 interception of a disappearing target. J Neurophysiol 118: 404-415, 2017.

720 de Lange FP, Heilbron M, Kok P. How Do Expectations Shape Perception? Trends Cogn Sci 22:

$721 \quad 764-779,2018$.

722 Lawrence MA. Easy analysis and visualization of factorial experiments [Online].

723 2016.http://github.com/mike-lawrence/ez.

724 Macavoy MG, Gottlieb JP, Bruce CJ. Smooth-pursuit eye movement representation in the 725 primate frontal eye field. Cereb Cortex 1: 95-102, 1991.

726 Madelain L, Krauzlis RJ. Pursuit of the ineffable: perceptual and motor reversals during the 727 tracking of apparent motion. J Vis 3: 642-653, 2003.

728 Mather G, Pavan A, Campana G, Casco C. The motion aftereffect reloaded. Trends Cogn. Sci. 729 12: 481-487, 2008. 
Maus GW, Potapchuk E, Watamaniuk SNJ, Heinen SJ. Different time scales of motion integration for anticipatory smooth pursuit and perceptual adaptation. $J$ Vis 15: 1-13, 2015.

Meier K, Giaschi D. The maturation of global motion perception depends on the spatial and temporal offsets of the stimulus. Vision Res 95: 61-67, 2014.

734 Missal M, Heinen SJ. Supplementary Eye Fields Stimulation Facilitates Anticipatory Pursuit. J 735 Neurophysiol 92: 1257-1262, 2004.

736 Montagnini A, Spering M, Masson GS. Predicting 2D Target Velocity Cannot Help 2D Motion 737 Integration for Smooth Pursuit Initiation. J Neurophysiol 96: 3545-3550, 2006.

738 Pasture C, Montagnini A, Perrinet LU. Humans adapt their anticipatory eye movements to the 739 volatility of visual motion properties. PLOS Comput Biol 16: e1007438, 2020.

740 Pelli DG. The VideoToolbox software for visual psychophysics: transforming numbers into 741 movies. Spat Vis 10: 437-42, 1997.

742 Pilly PK, Seitz AR. What a difference a parameter makes: A psychophysical comparison of 743 random dot motion algorithms. Vision Res 49: 1599-1612, 2009.

744 Prins N, Kingdom FAA. Applying the Model-Comparison Approach to Test Specific Research 745 Hypotheses in Psychophysical Research Using the Palamedes Toolbox. Front Psychol 9: 1250, 7462018.

747 Qian N, Andersen R, Adelson E. Transparent motion perception as detection of unbalanced 748 motion signals. I. Psychophysics. J Neurosci 14: 7357-7366, 1994.

749 Santos EM, Gnang EK, Kowler E. Anticipatory smooth eye movements with random-dot 750 kinematograms. J Vis 12: 1-1, 2012.

751 Santos EM, Kowler E. Anticipatory smooth pursuit eye movements evoked by probabilistic cues. 752 J Vis 17: 1-16, 2017. 
753 Schall JD. Visuomotor Functions in the Frontal Lobe. Annu Rev Vis Sci 1: 469-498, 2015.

754 Schütz AC, Braun DI, Gegenfurtner KR. Eye movements and perception: A selective review. $J$

755 Vis 11: 1-30, 2011.

756 Schütz AC, Braun DI, Movshon JA, Gegenfurtner KR. Does the noise matter? Effects of

757 different kinematogram types on smooth pursuit eye movements and perception. J Vis 10: 1-22,

$758 \quad 2010$.

759 Seriès P, Seitz AR. Learning what to expect (in visual perception). Front. Hum. Neurosci.

7607 7Frontiers Media S. A.: 668, 2013.

761 Spering M, Gegenfurtner KR. Contrast and Assimilation in Motion Perception and Smooth

762 Pursuit Eye Movements. J Neurophysiol 98: 1355-1363, 2007.

763 Spering M, Montagnini A. Do we track what we see? Common versus independent processing

764 for motion perception and smooth pursuit eye movements: A review. Vision Res 51: 836-852, 2011.

765 Team RC. R: A language and environment for statistical computing [Online]. R Foundation for

766 Statistical Computing2019.http://www.r-project.org/.

767 Thier P, Ilg UJ. The neural basis of smooth-pursuit eye movements. Curr Opin Neurobiol 15:

$768 \quad 645-652,2005$.

769 Watamaniuk SNJ, Bal J, Heinen SJ. A Subconscious Interaction between Fixation and

770 Anticipatory Pursuit. J Neurosci 37: 11424-11430, 2017.

771 Wei XX, Stocker AA. A Bayesian observer model constrained by efficient coding can explain

772 “anti-Bayesian” percepts. Nat Neurosci 18: 1509-1517, 2015.

773 Wu X, Rothwell AC, Spering M, Montagnini A. Comparing dynamic effects of expectation on

774 motion perception and pursuit eye movements. In: Program No. 226.12. 2019 Neuroscience

775 Meeting Planner. Chicago, IL, United States: Society for Neuroscience, 2019. 
776 Wu X, Rothwell AC, Spering M, Montagnini A. Opposite effects of expectation on motion

777 perception and anticipatory pursuit eye movements. J Vis 20: 567, 2020.

778 Yang J, Lee J, Lisberger SG. The interaction of Bayesian priors and sensory data and its neural

779 circuit implementation in visually guided movement. J Neurosci 32: 17632-17645, 2012. 